\title{
Capillary transport from barrel to clamshell droplets on conical fibers
}

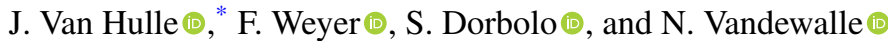 \\ CESAM-GRASP, Physics department B5a, University of Liège, B4000 Liège, Belgium
}

(Received 2 July 2020; accepted 22 January 2021; published 16 February 2021)

\begin{abstract}
Droplets spontaneously move when they are placed at the tip of a cone surface. Using three dimensionally printed structures, we experimentally explore a large panel of configurations regarding the aperture angle of the cone. We evidence a change of the droplet geometry while moving along the conical fiber. This transition is a switch of configuration from barrel to clamshell shape. The consequence is a change in the droplet dynamics. We estimate the position of this geometrical transition and we propose two models to describe the motion of the barrel and the clamshell droplets. While both shapes are driven by capillary forces, the dissipation is dependent on the geometrical configuration. For barrel shape droplets the main dissipation appears to be in the liquid wedge. For clamshell shape droplets the dissipation occurs mainly in the liquid film close to the conical fiber.
\end{abstract}

DOI: 10.1103/PhysRevFluids.6.024501

\section{INTRODUCTION}

In most arid regions like deserts, plants, and animals have developed specific strategies in order to survive long periods without rain [1]. Among others, one can cite the Phrynosoma cornutum lizard [2], the Physasterna cribripes darkling beetle [3], the Opuntia microdasys cactus [4], and the Syntrichia caninervis moss [5]. All the species have developed organs to harvest water: They have spikes, bumps, spines, or hairs that are able to collect tiny drops of water from air, fog, or rain. Mimicking those natural structures would lead to a promising way to supply water in arid regions. Large mesh nets have been designed to harvest water from fog [6,7]. Therefore, many studies focused on fog collection and tried to understand this complex process: From the fog flow through the mesh to the droplet impact to water collection [8-11]. The aim is to make each step as efficient as possible. Understanding the behavior of droplets on cylindrical fibers is also important to master water collection. Gilet et al. described droplets sliding on inclined cylindrical fibers [12] and Weyer et al. considered the conditions for a droplet to cross a node [13]. Also, in the static case, the angle of a bent fiber that can hold a maximal amount of water is known [14]. Besides that, there is another way to achieve fog harvesting: conical fibers [15-18] that are directly inspired from cactus spines. Although cylindrical fibers have been largely studied, conical fibers is a flourishing subject.

Recent works [19-21] have proposed that spine shapes could trigger the motion of droplets toward the plant itself. Indeed, a droplet on a conical fiber is known to move toward large radius, as it can be seen in Fig. 1, due to the imbalance between surface tension forces acting on the droplet surface. This effect was first characterized by Lorenceau et al. [19]. They studied this phenomenon for specific materials and scales, namely microliter silicone oil droplets on prewetted conical copper wires with a half-angle $\alpha \approx 0.38^{\circ}$ and a length $L=3 \mathrm{~cm}$. Those cones were created by pulling a cylindrical copper wire out of an acid bath. Other studies used different ways to manufacture conical fibers like pulling glass [22-25], using an electrochemical corrosion gradient [26] or even polishing brass [27].

\footnotetext{
*jvanhulle@uliege.be
} 


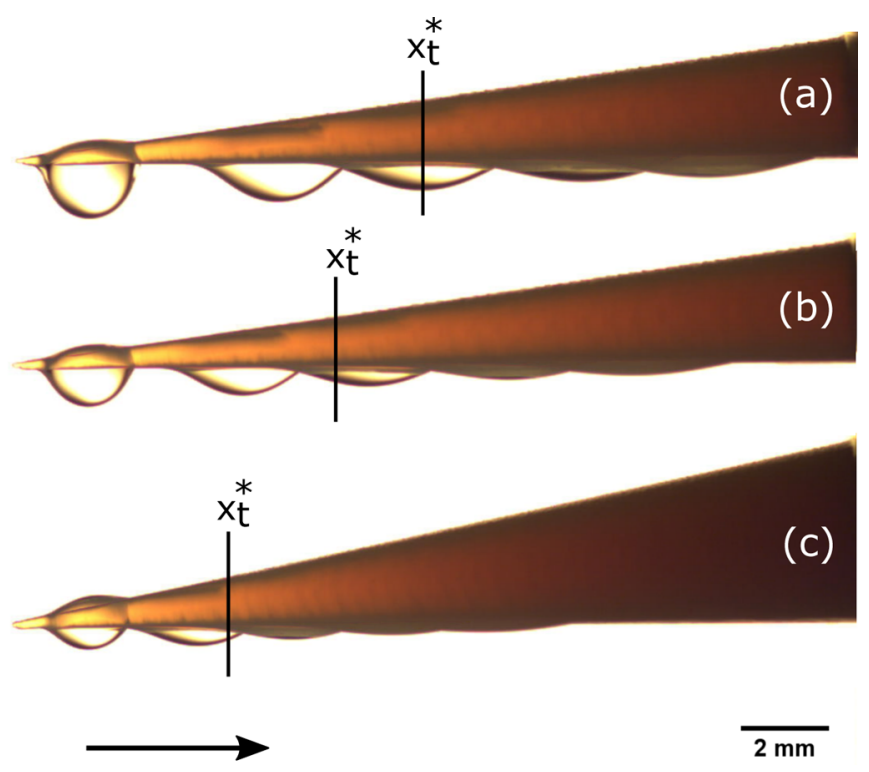

FIG. 1. Superposition of successive pictures emphasizing the motion of a droplet on a 3D-printed conical structure. The motion is shown at time $t=0 \mathrm{~s}, 2 \mathrm{~s}, 7 \mathrm{~s}, 20 \mathrm{~s}$, and $45 \mathrm{~s}$. (a) $\alpha=4^{\circ}, \Omega=4 \mu \mathrm{l}$; (b) $\alpha=4^{\circ}$, $\Omega=2 \mu \mathrm{l}$; and (c) $\alpha=6^{\circ}, \Omega=2 \mu \mathrm{l}$. The vertical black line is the estimated position of transition $x_{t}^{*}$ which is the distance after which the droplet should switch from the barrel shape to the clamshell shape. This value is calculated from Eq. (2).

According to the relative size of the fiber and of the droplet, the droplet on cylindrical and conical fibers can present two distinct geometries: barrel or clamshell [28]. A droplet with a barrel shape fully wraps the section of the fiber while a droplet in a clamshell shape covers only one side of the fiber. In all experimental studies listed above, the droplets adopt a barrel shape, except for two studies which focus on droplets showing a clamshell shape [24,27]. The influence of gravity on the droplet shape is neglected in their models because its size is below the capillary length. Note also that all those previous studies used prewetted conical fibers. A table comparing the experimental parameters used in this study to the recent publications is presented in Table I in order to position our work.

TABLE I. Table comparing previous studies of droplets moving along conical fibers. This table shows the experimental parameters used, namely half-angles $\alpha$ of the apex and the length $L$ of the conical fiber. The studies are categorized by the shape adopted by the droplet on those fibers, either barrel or clamshell shape. The present work is placed in another category as both barrel and clamshell shapes are experimentally observed on a single conical fiber.

\begin{tabular}{llcc}
\hline \hline & & Half-angle $\alpha\left({ }^{\circ}\right)$ & Cone length $L(\mathrm{~cm})$ \\
\hline Barrel & Lorenceau [19] & 0.38 & 3.0 \\
& Li [22] & 0.89 & 0.4 \\
& Michielsen [23] & $10.5,14.2,16$ & 1.0 \\
\multirow{3}{*}{ Clam } & Lee [25] & $\approx 0.05$ & $\approx 0.5$ \\
& Lv [24] & 3.2 & 1.0 \\
Barrel \& Clam & McCarthy [27] & $5,10,20,45$ & 1.5 \\
\hline \hline
\end{tabular}


The major motivation of this work is to achieve an extensive study on capillary transport of droplets lying on larger dry conical structures. Moreover, as can be seen in Table I, we use conical fibers with a wide variety of large half-angles and with a relatively long length compared to other studies. We observe that such fibers allow droplets transportation. Figure 1 shows a superposition of pictures emphasizing the motion of large droplets in three different cases that will be discussed in Sec. III. In all the situations shown in Fig. 1, the droplet spontaneously moves from the tip to the base of the cone. In addition to this directional motion, a shape transition of the droplet is observed during this motion. The droplet switches from the barrel shape to the clamshell shape. Previous experimental studies focused on either the barrel shape or the clamshell shape but none has experimentally observed this change of geometry (without any wetting gradient). We were able to witness this transition of shapes thanks to the properties of the cones we use (dry cones, long length, large half-angles). In this work, we propose to perform an experimental study of this motion driven by capillarity, as well as by the geometry switch. Our results allow us to estimate the position of the geometrical transition and to propose two models for macroscopic capillary transport of both the barrel and the clamshell shape droplets on conical fibers.

\section{METHODS}

In order to make an extensive experimental study, we three-dimensionally (3D) printed several conical structures using Object Prime 30 from Stratasys. The polymer used by the printer is acrylonitrile butadiene styrene (ABS). This 3D-printing technology offers an easy and controllable way of manufacturing cones. On top of it, the flexibility of the 3D-printing technique offers the opportunity to explore various cone angles. We considered half-angles $\alpha$ from $3^{\circ}$ to $10^{\circ}$. Those cones were slightly inclined for the experiment: The cone axis is tilted with the angle $\alpha$ in order to ensure that the lowest part of the cone was horizontal. Therefore, the droplet motion cannot be only attributed to gravity effects.

Prior to each experiment, we cleaned the cones with isopropanol and water and let them dry. The droplet was dropped off on the cone's tip thanks to a micropipette (Eppendorf Xplorer). We used silicone oil (Dow Corning), with a viscosity $v=50 \mathrm{cSt}$. The static contact angle between silicone oil and ABS is $\theta \approx 0^{\circ}$. Therefore, we were in the case of total wetting. We chose to focus on a range of droplet volumes $\Omega$ from 2 to $4 \mu \mathrm{l}$. Higher volumes could not be tested because such larger droplets drop off the fiber (in particular near the tip).

Perpendicular to the cone's axis of symmetry, a CCD (charge-coupled device) camera recorded the motion of the droplet (at $20 \mathrm{fps}$ ). A white background was lighted to achieve a good contrast. By image treatment, the left and right positions $x_{-}$and $x_{+}$of the droplet from the apex were determined. The position of the droplet center is defined by $x=\left(x_{+}+x_{-}\right) / 2$, as shown in the inset of Fig. 2 .

\section{RESULTS}

The typical evolution of the droplet position over time is shown in Fig. 2. This shows that the position $x$ increases, at first rapidly and then more slowly. Moreover, we plot the position over time for different half-angles $\alpha$ and different volumes $\Omega$ in order to highlight the influence of both parameters on the droplet motion. For the same interval of time, the droplet with the largest volume, moving on the cone with the smallest half-angle, goes further than the other ones.

In our experiments, the motion of the droplet is characterized by the capillary number $\mathrm{Ca}=$ $\dot{x} v \rho / \gamma \approx 2 \times 10^{-3}$, where $\dot{x}$ is the droplet speed, $\gamma$ is the surface tension, and $\rho$ is the density. This value indicates that the surface tension force overcome viscous one. The Weber number can also be calculated $\mathrm{We}=\rho \dot{x}^{2} L / \gamma \approx 7 \times 10^{-5}$, where $L$ is the characteristic length of the system. Here $L$ is the typical diameter of a droplet: $L \approx 1.5 \times 10^{-3} \mathrm{~m}$. It tells us that surface tension effects also dominate inertial forces. The Reynolds number, defined as $\operatorname{Re}=\dot{x} L / v$, compares inertial forces to viscous forces. For our experiments, its value is $\mathrm{Re} \approx 0.03$, therefore viscous forces are larger than inertial ones. The Bond number Bo is the ratio of gravitational forces to capillary forces, Bo $=$ 


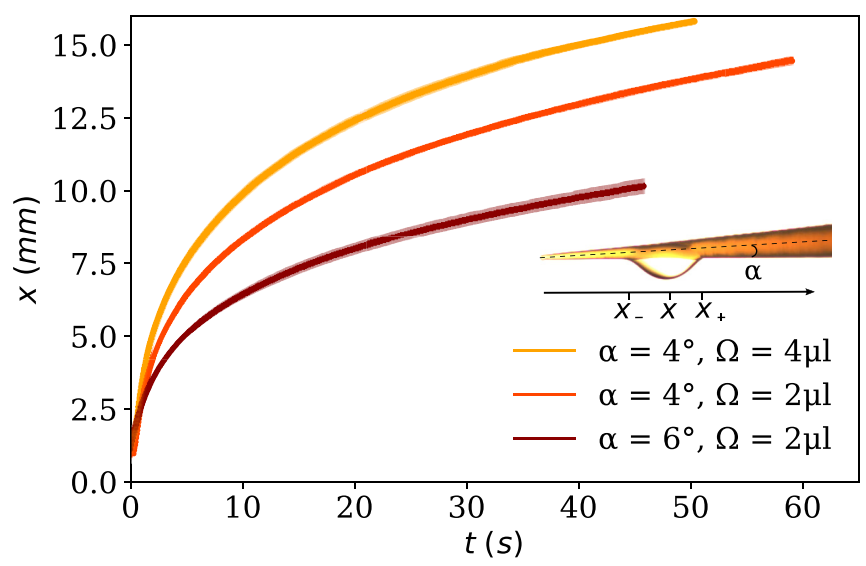

FIG. 2. Position from the apex of the droplet center $x$ along a cone as a function of time $t$. Different half-angles and volumes are shown: $\alpha=4^{\circ}, \Omega=4 \mu \mathrm{l} ; \alpha=4^{\circ}, \Omega=2 \mu \mathrm{l}$; and $\alpha=6^{\circ}, \Omega=2 \mu \mathrm{l}$. The error bars coming from repeated experiments for each data set are in light color. The inset defines the half-angle $\alpha$ of the cone as well as the position from the apex of the droplet center defined by $x=\left(x_{+}+x_{-}\right) / 2$.

$\rho g L^{2} / \gamma \approx 1.04$, where $g$ is the gravitational acceleration. It reveals that gravity affects the barrel shape droplets.

To evidence the droplet motion, we compute the speed of the droplet $\dot{x}$ as a function of the inverse of position $1 / x$. This speed is obtained using a five-point derivative of the data $x(t)$ from Fig. 2 . This is illustrated with a double logarithmic graph in Fig. 3 for the same cases as presented in Fig. 2. The speed decreases as the position on the fiber increases, i.e., as the inverse position decreases. The three curves clearly indicate that the motion is affected by the half-angle $\alpha$ and the volume $\Omega$. Furthermore, it can be seen that as the half-angle increases, the speed decreases. Changing the volume reveals that the higher the volume is, the higher the speed is.

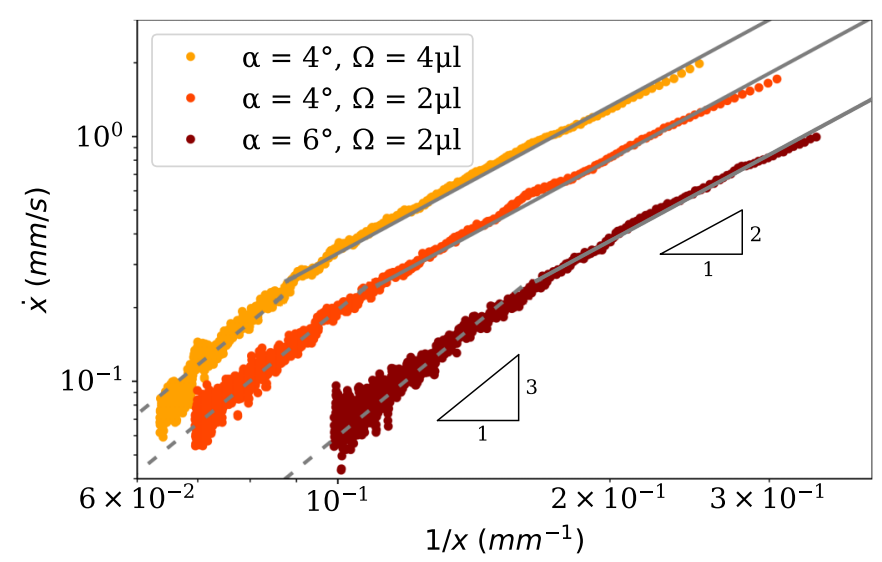

FIG. 3. Double logarithmic graph of the speed $\dot{x}$ of the droplet as a function of the inverse position $1 / x$, for different half-angles and volumes: $\alpha=4^{\circ}, \Omega=4 \mu \mathrm{l} ; \alpha=4^{\circ}, \Omega=2 \mu \mathrm{l}$; and $\alpha=6^{\circ}, \Omega=2 \mu \mathrm{l}$. The short vertical colored lines are the inverse of the transition position $1 / x_{t}^{*}$ [Eq. (2)]. The grey lines are fitting curves corresponding to the barrel shape model [Eq. (11)], straight line, and to the clamshell model [Eq. (12)], dashed line. 


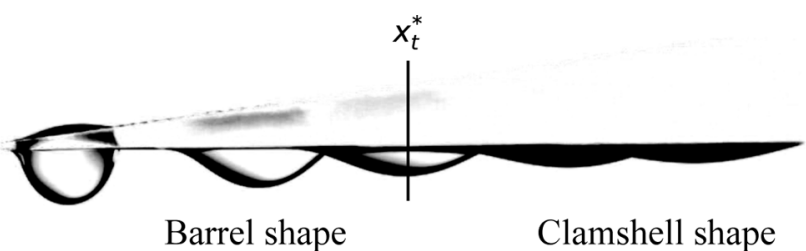

FIG. 4. Image treatment of the superposition shown in Fig. 1(a). This image treatment, which removes the image of the cone without any droplet, allows us to highlight the geometry of the droplet and the contact area of the droplet with the cone as well as the contact line. We tuned the contrast to emphasize the top liquid film. The droplet position along the cone is shown at time $t=0 \mathrm{~s}, 2 \mathrm{~s}, 7 \mathrm{~s}, 20 \mathrm{~s}$, and $45 \mathrm{~s}$ and the experimental parameters are $\alpha=4^{\circ}$ and $\Omega=4 \mu$ l. One can see that the droplet has a barrel shape close to the tip of the cone and then, as the droplet gets closer to the base, we see a change in geometry from a barrel to a clamshell shape. The vertical black line is the position of transition $x_{t}^{*}$ calculated from Eq. (2).

\section{DISCUSSION}

In order to describe the dynamics of the droplet, we have to inspect the geometry of the system. Although both position and speed are smooth curves, the droplet is submitted to a major change of geometry, as it can be seen from Fig. 4. This image is obtained using ImageJ [29] and is the result of the difference between Fig. 1(b) and the picture of the cone without any droplet. Consequently, the geometry of the droplet is highlighted. We tuned the contrast of the picture to emphasize the top liquid film. Close to the tip of the cone, the droplet has a barrel shape, i.e., wraps the cone, with a deformation due to gravity (the droplet has a thin film above the cone and a hanging part under the cone). As the droplet moves, the droplet switches to a clamshell shape: The droplet does not wrap the cone any longer but sits on the bottom of it. Whatever the shape of the droplet, it moves toward the base of the cone. The change of geometry is the opposite of a roll-up transition $[28,30,31]$. It occurs on a fiber when the volume of the droplet is reduced or the radius of the fiber is getting larger. Here we observe this transition with a constant droplet volume and on a fiber that has a conical shape. Thus, as the droplet moves, the local radius of the fiber increases. In the case of cylindrical fibers, an adimensional number, called the reduced volume $V^{*}$, has been introduced to predict the transition from one shape to the other [28]. It is the ratio between the volume of the droplet and the cube of the fiber radius and is defined as

$$
V^{*}=\frac{\Omega}{r^{3}}
$$

If this number is large, then the droplet is expected to adopt a barrel shape and if this number is small, then the droplet should have a clamshell shape. In our case, we can use this adimensional number to estimate the position where the transition between barrel and clamshell shapes occurs. Let us note $V_{t}^{*}$ the reduced volume at the transition. We can then obtain the transition position given by

$$
x_{t}^{*}=\frac{1}{\sin \alpha} \sqrt[3]{\frac{\Omega}{V_{t}^{*}}} .
$$

Below this transition position, the shape adopted by the droplet should be a barrel and beyond, the droplet should switch to a clamshell shape. Experimentally, it is difficult to track the contact line because the droplet is transparent and the shape transition is a smooth process. Therefore, we can experimentally approximate the range of positions where the switch in shape occurs. We found an empirical value for the reduced volume at the transition, $V_{t}^{*}=8$. This value is kept constant for all the post-treatment of our data. In Sec. IV C there is a discussion about how this empirical value is obtained. The transition positions are denoted in Figs. 1, 3, and 4 with vertical lines. 


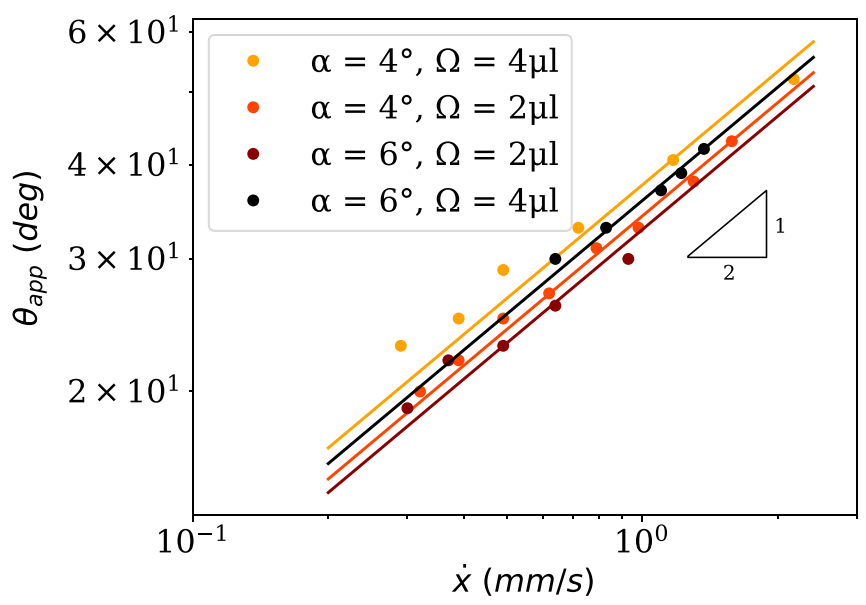

FIG. 5. Double logarithmic graph of the apparent contact angle of barrel shape droplets $\theta_{\text {app }}$ as a function of the droplet speed $\dot{x}$, for different half-angles and volumes: $\alpha=4^{\circ}, \Omega=4 \mu \mathrm{l} ; \alpha=4^{\circ}, \Omega=2 \mu \mathrm{l} ; \alpha=6^{\circ}$, $\Omega=4 \mu 1$; and $\alpha=6^{\circ}, \Omega=2 \mu \mathrm{l}$. The experimental data points are fitted with a power law with exponent $1 / 2$.

This shape transition is in good agreement with the simulations made by Liang et al. [21] using Surface Evolver [32]. They also observed, in their simulations, a transition from a barrel shape to a clamshell shape (without gravity) and, whatever the shape, the droplet goes toward large radius of curvature.

In order to explain these observations, a model taking into account the balance of the forces exerted on the droplet is developed. The droplet experiences two types of forces: the capillary force and the viscous force. In our problem, we have two geometrical configurations, and therefore we have to describe both cases independently.

\section{A. Barrel shape}

Most of the previous studies consider the motion of axisymmetrical barrel shape droplets moving on conical substrates. The models are based on a driving force coming from a gradient of the Laplace pressure along the surface of the droplet $[19,22]$. In our case, the barrel shape is not axisymmetrical due to gravitational effects. Finding the gradient of Laplace pressure in such a nonsymmetrical geometry is a significant problem. This is why we use, for the driving force, the external force applying along the contact line of the droplet. This driving force has been used in the model of Lee [25] to describe the motion of submillimetric symmetrical barrel shape droplets on prewetted conical glass fibers. In opposition to Lee [25], we do not consider the two contact lines of the barrel as our conical fiber is not prewetted. In fact, in our case, the advancing contact line of the droplet is wetting a dry surface while, along the receding contact line, the surface is not dewet, and the droplet is leaving a thin film. Therefore, the wetting by the advancing contact line is the slower process leading the motion. This is why we only consider the wetting force along the advancing contact line as the main contribution to the driving force. Along the advancing contact line, one can see an apparent contact angle (see Fig. 1), we note it $\theta_{\text {app. }}$ This angle is maximum just after the release of the droplet. The angle decreases as the droplet spreads. The dynamics of the wedge angle of a droplet $\theta$ on a flat surface is given by the law of Tanner $\theta \approx(v \rho \Gamma \dot{x} / \gamma)^{1 / 3}$, where $\Gamma$ is a constant that takes the value 15 if liquid slides on a dry surface as showed by Hoffman [33]. Figure 5 shows a double logarithmic graph of the apparent contact angle as a function of the speed. We find a slope of $1 / 2$ instead of $1 / 3$ as the Tanner's law provides. Therefore, to characterize the dynamics of the apparent contact angle on a cone, we use this law: $\theta_{\text {app }} \approx(\nu \rho \Gamma \dot{x} / \gamma)^{1 / 2}$. The capillary length, $l_{c}$, describes the competition between capillary effects and gravitational effects. We 
(a)

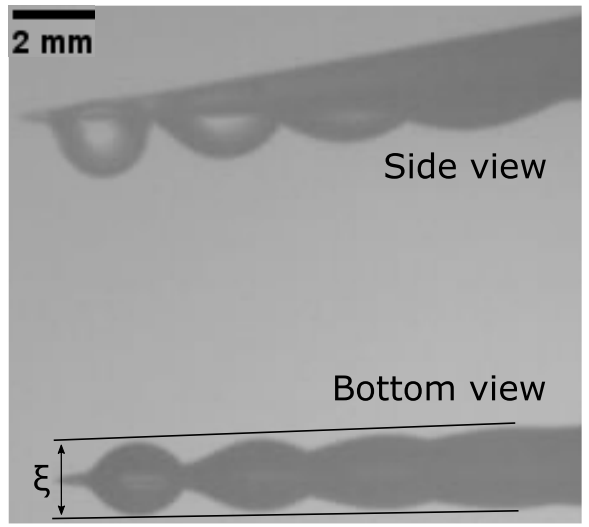

(b)

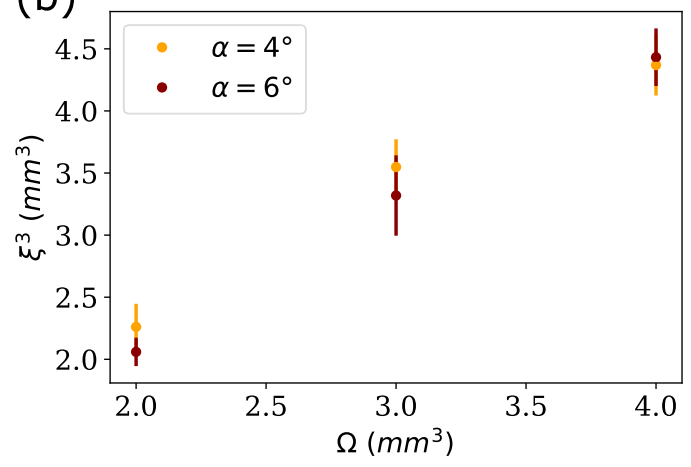

FIG. 6. (a) Superpositions of pictures of a droplet moving along the cone with two different views, side view and bottom view, taken simultaneously. With the bottom view one can see that the width $\xi$ of the barrel shape droplet is roughly constant. The experimental parameters are $\alpha=4^{\circ}$ and $\Omega=3 \mu \mathrm{l}$. (b) Graph of the width of the barrel shape droplet to the power three as a function of the volume of the droplet and for different half-angles. The width of the droplet can be scaled by the cube root of the volume.

have $l_{c}=\sqrt{\gamma /(\rho g)} \approx 1.510^{-3} \mathrm{~m}$. With a bottom view of the fiber, one observes that the width of the hanging part of the barrel shape droplet is roughly constant as shown in Fig. 6(a). Actually, it has the same order of magnitude as the capillary length. This width, noted $\xi$, can be scaled thanks to the volume of the droplet with the relation $\xi \approx \Omega^{1 / 3}$, as illustrated in Fig. 6(b). So we assume that the capillary force is acting on a length scale comparable to this characteristic length. This leads to a constant driving force given by

$$
F_{\gamma, \text { barrel }} \approx \gamma \Omega^{1 / 3}
$$

Now let us look at the expression of the viscous force. We manage to perform experiments with droplets full of fluorescent particles (Fluostar EBM, $15 \mu \mathrm{m}$ ) in order to see the motion of the liquid inside the droplet. A movie is available in the Supplemental Material [34], video 1. We observe a circulation of the fluorescent particles toward the advancing liquid wedge. Therefore we assume that the dissipation in the front liquid wedge is dominating. Lorenceau et al. [19] explained that the viscous stress is given by $\rho v \dot{x} r \Gamma / \theta$. For our system, one obtains the following dissipating force in the liquid wedge:

$$
F_{\text {wedge }} \approx \gamma x \sin \alpha\left(\frac{\nu \rho \Gamma \dot{x}}{\gamma}\right)^{1 / 2} .
$$

The balance of these forces acting on the droplet can be written as

$$
\gamma \Omega^{1 / 3}-\gamma x \sin \alpha\left(\frac{v \rho \Gamma \dot{x}}{\gamma}\right)^{1 / 2}=\rho \Omega \ddot{x}
$$

where $\ddot{x}$ is the acceleration of the droplet. We study the stationary equation that gives the speed limit of the droplet for each position along the cone. One obtains

$$
\dot{x} \approx \frac{\gamma}{v \rho \Gamma} \frac{\Omega^{2 / 3}}{(x \sin \alpha)^{2}} .
$$

The model for the barrel shape predicts a linear dependency between the speed and the square of the inverse position. In Fig. 3, one can observe a slope of two for large values of $1 / x$, namely when the droplet adopts a barrel shape. We will check the scaling in Sec. IV C. 


\section{B. Clamshell shape}

A droplet with a clamshell shape set only on one side of the fiber. That kind of droplet hanging under a cone has a nonsymmetrical front-rear contact line. If the droplet goes from the tip to the base of the cone, then the radius of curvature at the front of the droplet is larger than the one at the rear. The droplet with a clamshell shape is thin; it is a flat droplet. For the driving force we assume that it is the same expression than the one developed for thin symmetrical barrel shape droplets. We have

$$
F_{\gamma, \text { clam }} \approx \gamma \Omega /\left(x^{2} \sin \alpha\right)
$$

This expression comes from the Laplace law applied in the case of thin barrel shape droplets [19].

For the viscous force, we assume that the dissipation inside the liquid close to the fiber dominates. Indeed, as we can see in the movie in the Supplemental Material [34] video 1, when the droplet has a clamshell shape, the fluorescent particles are stretched in the fluid layers during the motion. This film dissipation is given by

$$
F_{\text {film }} \approx v \rho \Gamma \frac{e}{\omega} x \dot{x} \sin \alpha,
$$

where $e$ is the spread of the droplet $\left(e=x_{+}-x_{-}\right)$and $\omega$ is the thickness of the thin liquid film where the dissipation occurs [19]. For the description of the position over time for a droplet experiencing a film dissipation, we have

$$
\frac{\gamma}{\sin \alpha} \frac{\Omega}{x^{2}}-v \rho \Gamma \sin \alpha \frac{e}{\omega} x \dot{x}=\rho \Omega \ddot{x} .
$$

The stationary expression is therefore given by

$$
\dot{x} \approx \frac{\gamma}{v \rho \Gamma} \frac{\omega}{e} \frac{1}{\sin ^{2} \alpha} \frac{\Omega}{x^{3}} .
$$

This is the same model as the one developed by Lorenceau et al. to explain the motion of symmetrical barrel shape droplet. With the present description, we extend this model to the case of clamshell shape droplets. The model for the clamshell shape predicts a speed proportional to the cube of the inverse position, as can been seen in Fig. 3 (range of small values of $1 / x$ ). Also, the speed is linked to the droplet volume and the half-angle of the cone. We will check this scaling in the following subsection.

\section{Analysis of the models}

We have proposed in the previous sections two specific models for both regimes corresponding to different shapes. We now have to fix the range of $x$ corresponding to each geometry. Therefore, we need to estimate the transition position $x_{t}^{*}$. To find the empirical value of the reduced volume at the transition, we tune the boundary between the two regimes until the models fit best the data for both regimes. Then, we confirm the given position transition [Eq. (2)] by looking at the experimental movies and see that the found range is well approximated. Indeed, thanks to image treatments like the one illustrated in Fig. 4, we can see where the droplet has a barrel shape or a clamshell shape. Also, the reduced volume at the transition can be confirmed by the fact that the droplet should change its shape when the width of the droplet corresponds to the diameter of the fiber. Therefore, we have $r_{t}^{*} \approx \sqrt[3]{\Omega} / 2$, and with Eq. (1), we have $V_{t} *=\Omega / r_{t}^{* 3} \approx 8$. With that we obtain the range of positions corresponding to the barrel shape and to the clamshell shape. We can fit the data of the speed as a function of the inverse position for both regions with the corresponding model.

For the barrel shape, the model predicts that the speed goes as the square of the inverse position [Eq. (6)]. The proportionality coefficient is the fitting parameter noted $a_{b}$. We have,

$$
\dot{x}=\frac{a_{b}}{x^{2}} .
$$




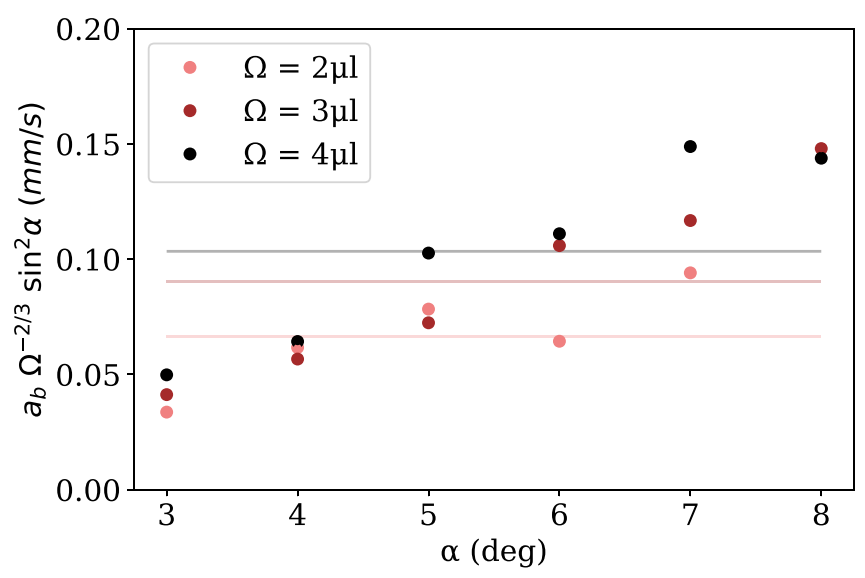

FIG. 7. Graph of $a_{b} \Omega^{-2 / 3} \sin ^{2} \alpha$, where $a_{b}$ is the fitting parameter for the barrel shape description, as a function of the half-angle and for different volumes. This parameter $a_{b}$ represents the proportionality factor between the speed and the inverse of position to the power two [Eq. (11)]. Therefore, the higher it is, the higher the speed is. One can see that it increases with the droplet volume and decreases with the half-angle of the cone. The model overestimates the influence of the half-angle. The error bars are smaller than the size of the symbols. The horizontal lines corresponds to an average.

This fitting law is plotted with a straight gray line in Fig. 3, we see that it is in good agreement with our experimental data. The fits are in excellent agreement for all our data except for the halfangle $\alpha=3^{\circ}$ (determination coefficient of those fits are $r^{2} \approx 0.94$ ). In Fig. 7, we have plotted $a_{b} \Omega^{-2 / 3} \sin ^{2} \alpha$ as a function of $\alpha$. We have $a_{b} \Omega^{-2 / 3} \sin ^{2} \alpha \approx 0.08 \mathrm{~mm} / \mathrm{s}$. According to Eq. (6), this expression can also be estimated with $\gamma /(\nu \rho \Gamma) \approx 0.03 \mathrm{~mm} / \mathrm{s}$, which is the same order of magnitude. In Fig. 7, one observes that the ratio $a_{b} \Omega^{-2 / 3} \sin ^{2} \alpha$ is independent of the volume. The influence of the volume is well described by the model. One can also observe that this ratio also increases with the half-angle. This highlights a limitation of the model, it overestimates the influence of the half-angle. Different reasons could explain this observation. The capillary force acts mainly where the apparent contact angle is the largest and we have scaled this length with the width of the droplet. The barrel shape has a symmetrical shape neither rear-front nor top-bottom. Moreover, the advancing contact line is inclined as the droplet is moving. A more precise description of the shape and the length of the contact line should provide a better scaling for the half-angle of the cone.

For the clamshell shape, the model predicts that the speed goes as the cube of the inverse of the position [Eq. (10)]. We note the proportionality coefficient $a_{c}$ and it is the fitting parameter. We obtain

$$
\dot{x}=\frac{a_{c}}{x^{3}} .
$$

This fitting law is plotted with a dashed gray line in Fig. 3. Our model for the clamshell shape seems to depict the good trend of our experimental data. On the graph presented in Fig. 8, showing $a_{c} \sin ^{2} \alpha$ as a function of the half-angle, one can see that the speed of a droplet with a clamshell shape decreases with the half-angle and increases with the volume, as predicted by our model. By looking at the expression of the speed [Eq. (10)], one can see that the speed tends to zero for very large positions. It is known that there is no equilibrium position for the clamshell shape if no external forces apply (gravity or contact angle hysteresis) [27,35]. We reinforce this result with our observations. With the fitting parameter $a_{c}$, the ratio $\omega / e$ can be calculated. We have $\omega / e \approx$ $a_{c} v \rho \Gamma \sin ^{2} \alpha /(\gamma \Omega) \approx 10^{-2} \mathrm{~m}$. It is a realistic order of magnitude because the spread is $e \approx 10^{-3} \mathrm{~m}$ and $\omega$ is the thickness of the layers of fluid where film dissipation occurs, having $\omega \approx 10^{-5} \mathrm{~m}$ is acceptable. 


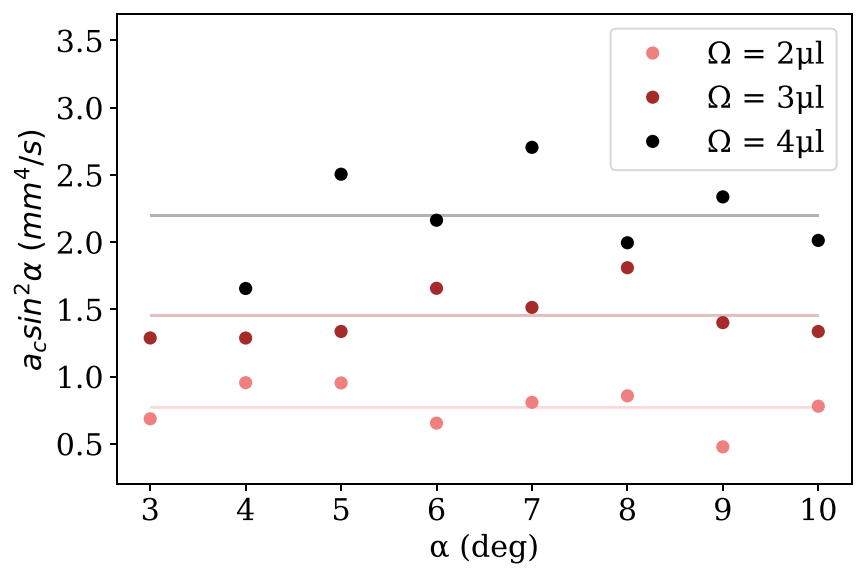

FIG. 8. Graph of $a_{c} \sin ^{2} \alpha$, where $a_{c}$ is the fitting parameter for the clamshell shape description, as a function of the half-angle and for different volumes. This parameter $a_{c}$ represents the slope of the speed as a function of the cube of the inverse position [Eq. (12)]. Therefore, the higher this coefficient is, the higher the speed is. The speed of a clamshell shape droplet increases with the droplet volume and decreases with the half-angle of the cone. The error bars are smaller than the size of the symbols. The horizontal lines corresponds to an average and gives the trend expected by the model.

\section{SUMMARY}

Conical fibers are manufactured in a highly controllable way using 3D-printing techniques. When a droplet is placed on those synthetic cones, a motion of the droplet is observed from the tip toward the base of the cone. Beyond the capillary length, the shape of the barrel shape droplet is influenced by the gravity. We have observed a switch in the geometry of the droplet. This transition is witnessed thanks to the use of dry cones (so that a clamshell shape can be defined) and thanks to a sufficiently long cone or a large half-angle so the position transition can be reached. For small radii, the droplet tends to adopt a barrel shape (deformed by gravity, meaning we have a thin film above the cone and a hanging part under the cone), but if the radius of the fiber is larger, the droplet adopts a clamshell shape. The two different shapes induce two different dynamics. These dynamics are explained with two separate models based on the balance of forces exerted on the droplet. For the barrel shape, the dissipation in the wedge dominates. A study of the evolution of the apparent contact with the speed of the droplet allows to adapt the Tanner's law for conical fibers. For the driving force, as the barrel shape is not symmetrical, an external capillary force is developed. For the clamshell shape, the dissipation in the thin film layers close to the fiber dominates. The driving force used is the Laplace law applied on thin barrel drops, this expression has been developed by Lorenceau et al. [19]. We actually obtain the same model as Lorenceau et al. They developed it to explain the motion of symmetrical barrel shape droplets but we extend it to the case of clamshell shape droplets. If the volume of the droplet is higher, then the speed of the droplet is higher too, for both the barrel shape and the clamshell shape droplets. The smaller the half-angle of the cone, the higher the speed for both barrel and clamshell shapes. Note that some experimental dependencies in the barrel shape model are yet to be described. In conclusion, capillary transport is enhanced if the volume of the droplet is large and if the half-angle of the cone is small.

The perspectives of this work are numerous. We could change the surface roughness of the cones by adding grooves, scales or spikes which could be done thanks to 3D printing. Indeed, roughnesses are observed on cactus spines, such as the Opuntia microdasys cactus, but their influence on capillary transport are not yet described. Another perspective of conical fibers is fog harvesting. We could perform experiments of fog collection with our new synthetic 3D-printed conical fibers and compare their efficiency with the one of real cactus spines. Another way to set a droplet into motion is 
to put it into a wedge made of two smooth surfaces [36,37]. It would be interesting to consider curved surfaces and to see if it changes the behavior of the droplet. Duprat et al. [38] described the shape of droplets trapped between two parallel cylindrical fibers. Replacing these fibers with conical ones could provoke the motion of the droplets. In conclusion, our study provides a new way for manipulating droplets and offers many new perspectives.

\section{ACKNOWLEDGMENTS}

S.D. acknowledges support as a F.R.S.-FNRS research associate. This work was partially supported by the FRS-FNRS (Brussels, Belgium) under Grant No. WISD X.3047.17 - PDR.

[1] F. T. Malik, R. M. Clement, D. T. Gethin, W. Krawszik, and A. R. Parker, Nature's moisture harvesters: a comparative review, Bioinspir. Biomim. 9, 031002 (2014).

[2] P. Comanns, G. Buchberge, A. Buchsbaum, B. Baumgartner, A. Logler, S. Bauer, and W. Baumgartne, Directional, passive liquid transport: the Texas horned lizard as a model for a biomimetic 'liquid diode', J. R. Soc. Interface 12, 20150415 (2015).

[3] T. Nørgaard and M. Dacke, Fog-basking behaviour and water collection efficiency in Namib Desert Darkling beetles, Front. Zool. 7, 23 (2010).

[4] J. Ju, H. Bai, Y. Zheng, T. Zhao, R. Fang, and L. Jiang, A multi-structural and multi-functional integrated fog collection system in cactus, Nat. Commun. 3, 1247 (2012).

[5] Z. Pan, W. G. Pitt, Y. Zhang, N. Wu, Y. Tao, and T. T. Truscott, The upside-down water collection system of Syntrichia caninervis, Nat. Plants 2, 16076 (2016).

[6] G. A. Al-hassan, Fog water collection evaluation in Asir region-Saudi Arabia, Water Resourc. Manage. 23, 2805 (2009).

[7] M. J. Estrela, J. A. Valiente, D. Corell, and M. M. Millàn, Fog collection in the western Mediterranean basin (Valencia region, Spain), Atmos. Res. 87, 324 (2008).

[8] J. de Dios Rivera, Aerodynamic collection efficiency of fog water collectors, Atmos. Res. 102, 335 (2011).

[9] K. C. Park anf S. S. Chhatre, S. Srinivasan, R. E. Cohen, and G. H. McKinley, Optimal design of permeable fiber network structures for fog harvesting, Langmuir 29, 13269 (2013).

[10] W. Shi, M. J. Anderson, J. B. Tulkoff, B. S. Kennedy, and J. B. Boreyko, Fog harvesting with harps, ACS Appl. Mater. Interfaces 10, 11979 (2018).

[11] M. Azeem et al., Optimal design of multilayer fog collectors, ACS Appl. Mater. Interfaces 12, 7736 (2020).

[12] T. Gilet, D. Terwagne, and N. Vandewalle, Droplets sliding on fibres, Eur. Phys. J. E. 31, 253 (2010).

[13] F. Weyer, A. Duchesne, and N. Vandewalle, Switching behavior of droplets crossing nodes on a fiber network, Sci. Rep. 7, 13309 (2017).

[14] Z. Pan, F. Weyer, W. G. Pitt, N. Vandewalle, and T. T. Truscott, Drop on a bent fibre, Soft Matter 14, 3724 (2018).

[15] K. Li, J. Ju, J. M. Z. Xue, L. Feng, S. Gao, and L. Jiang, Structured cone arrays for continuous and effective collection of micron-sized oil droplets from water, Nat. Commun. 4, 2276 (2013).

[16] M. Cao, J. Ju, K. Li, S. Dou, K. Liu, and L. Jiang, Facile and large-scale fabrication of a cactus-inspired continuous fog collector, Adv. Funct. Mater. 24, 3235 (2014).

[17] D. Gurera and B. Bhushan, Optimization of bioinspired conical surfaces for water collection from fog, J. Colloid Interface Sci. 551, 26 (2019).

[18] J. Ju, K. Xiao, H. Bai, and L. Jiang, Bioinspired conical copper wire with gradient wettability for continuous and efficient fog collection, Adv. Mater. 25, 5937 (2013).

[19] E. Lorenceau and D. Quéré, Drops on a conical wire, J. Fluid Mech. 510, 29 (2004).

[20] C. Luo, Theoretical exploration of barrel-shaped drops on cactus spines, Langmuir 31, 11809 (2015). 
[21] Y. E. Liang, H. K. Tsao, and Y. J. Sheng, Drops on hydrophilic conical fibers: Gravity effect and coexistent states, Langmuir 31, 1704 (2015).

[22] E. Q. Li and S. T. Thoroddsen, The fastest drop climbing on a wet conical fibre, Phys. Fluids. 25, 052105 (2013).

[23] S. Michielsen, J. Zhang, J. Du, and H. J. Lee, Gibbs free energy of liquid drops on conical fibers, Langmuir 27, 11867 (2011).

[24] C. Lv, C. Chen, Y. C. Chuang, F. G. Tseng, Y. Yin, F. Grey, and Q. Zheng, Substrate Curvature Gradient Drives Rapid Droplet Motion, Phys. Rev. Lett. 113, 026101 (2014).

[25] C. L. Lee, R. D. Schulman, E. Raphaël, and K. Dalnoki-Veress, Droplet migration on conical fibers arXiv:2008.13390.

[26] X. Tan, T. Shi, Z. Tang, B. Sun, L. Du, Z. Peng, and G. Liao, Investigation of fog collection on cactusinspired structures, J. Bionic Eng. 13, 364 (2016).

[27] J. McCarthy, D. Vella, and A. A. Castrejón-Pita, Dynamics of droplets on cones: Self-propulsion due to curvature gradients, Soft Matter 15, 9997 (2019).

[28] G. McHale, M. I. Newton, and B. J. Carroll, The shape and stability of small liquid drops on fibers, Oil Gas Sci. Technol. 56, 47 (2001).

[29] C.A. Schneider, W.S. Rasband, and K.W. Eliceiri, NIH Image to ImageJ: 25 years of image analysis, Nat. Methods 9, 671 (2012).

[30] B. J. Carroll, Equilibrium conformations of liquid drops on thin cylinders under forces of capillarity. A theory for the roll-up process, Langmuir 2, 248 (1986).

[31] G. McHale and M. I. Newton, Global geometry and the equilibrium shapes of liquid drops on fibers, Colloid. Surface 206, 79 (2002).

[32] K.A. Brakke, The surface evolver, Exp. Math. 1, 141 (1992).

[33] R. L. Hoffman, A study of the advancing interface, J. Colloid Interface Sci. 50, 228 (1975).

[34] See Supplemental Material at http://link.aps.org/supplemental/10.1103/PhysRevFluids.6.024501 for experimental movie of a droplet containing fluorescent particles moving along a conical fiber.

[35] Y. Li, H. Wu, and F. Wang, Stagnation of a droplet on a conical substrate determined by the critical curvature ratio, J. Phys. D: Appl. Phys. 49, 085304 (2016).

[36] E. Ruiz-Gutiérrez, J. H. Guan, B. Xu, G. McHale, G. G. Wells, and R. Ledesma-Aguilar, Energy Invariance in Capillary Systems, Phys. Rev. Lett. 118, 218003 (2017).

[37] E. Reyssat, Drops and bubbles in wedges, J. Fluid. Mech. 748, 641 (2014).

[38] C. Duprat, S. Protière, A. Y. Beebe, and H. A. Stone, Wetting of flexible fibre arrays, Nature 481, 510 (2012). 